\title{
Ultrasonic Measurement for the Experimental Investigation of Velocity Distribution in Vapor-Liquid Boiling Bubbly Flow
}

\author{
Wongsakorn Wongsaroj ${ }^{1, *}$, Hideharu Takahashi ${ }^{2}$, Natee Thong-Un ${ }^{1}$, Hiroshige Kikura ${ }^{2}$ \\ ${ }^{1}$ Department of Instrumentation and Electronics Engineering, King Mongkut's University of Technology North Bangkok, \\ Bangkok, Thailand \\ ${ }^{2}$ Laboratory for Zero-Carbon Energy, Tokyo Institute of Technology, Tokyo, Japan \\ Received 22 August 2021; received in revised form 23 October 2021; accepted 24 October 2021
}

DOI: https://doi.org/10.46604/ijeti.2021.8329

\begin{abstract}
This study proposes an ultrasonic velocity profiler (UVP) with a single ultrasonic gas-liquid two-phase separation (SUTS) technique to measure the velocity distribution of vapor-liquid boiling bubbly flow. The proposed technique is capable of measuring the velocity of the vapor bubble and liquid separately in boiling conditions. To confirm the viability of the measurement technique, the experiment is conducted on vertical pipe flow apparatus. The ultrasonic transmission and effect of ultrasonic refraction through the pipe wall and water are investigated at ambient temperature until subcooled boiling temperature is reached. The velocity profile in the water at elevated temperature is measured to verify the ability of the technique in this application. The bubbly flow velocity distribution measurement in boiling conditions is then demonstrated. The results show that the proposed technique can effectively investigate the velocity of both phases under various fluid conditions in boiling bubbly flow.
\end{abstract}

Keywords: ultrasonic, velocity, boiling, vapor, bubbly

\section{Introduction}

Boiling two-phase flow is a heat transfer phenomenon and plays an important role in the heat transfer process for many industries. Flow boiling heat transfer has high cooling efficiency and is used in various applications, including boiler tubes, evaporators, chemical processes, and the cooling of nuclear reactors (such as boiling water reactors (BWRs)). It is utilized to prevent the problem of high heat flux dissipation. In the subcooled boiling region, until fully boiling, vapor-liquid two-phase bubbly flow is formed and directly affects the performance and safety of the heat exchange channel. The flow behavior in this region affects downstream boiling and the characteristics existing downstream of the channel. The flow in this region can also be responsible for developing flow instabilities in the heat exchange channel. Lastly, heat transfer enhancement is influenced by the vapor-liquid boiling two-phase flow that occurs [1-2]. Numerical analysis is utilized to assess the efficiency and safety level of the heat exchange system [3-4].

To obtain the correlation model used in the analysis, the experimental data from the vapor bubble and liquid phases are required, such as bubble diameter and void fraction. In particular, the velocity value of both phases is a key factor in the constitutive equation. This parameter strongly affects heat transfer enhancement [5] and phase distribution [6], which involves the performance and safety of the heat exchange channel, especially in BWRs. Therefore, the experimental investigation of velocity distribution in vapor-liquid boiling bubbly flow is crucial. The velocity data of both phases must be obtained separately.

* Corresponding author. E-mail address: wongsakorn.w@eng.kmutnb.ac.th

Tel.: +66-2-5552000 ext 8641; Fax: +66-2-5874846 
Intrusive techniques have been applied to investigate the flow velocity in bubbly flow, such as conductive probes [7]. These methods involve direct contact with the fluid, increasing the risk of instrument damage. Non-intrusive measurement techniques that can measure the velocity data in both phases have been proposed, such as particle image velocimetry (PIV) [8]. However, PIV fails if the working fluid is opaque. Furthermore, the PIV measurement at the channel behind the bubble will have low accuracy due to the weakness of the laser sheet. The X-ray method can overcome most PIV drawbacks but requires a specific configuration and procedure [9]. Moreover, the X-ray method is mainly carried out on either single or a few bubbles to avoid the bubble overlapping in the flow images, which is the main disadvantage. Therefore, a measurement technique that is "non-intrusive", "works on less transparent fluid", and applicable for the "bubble overlapping condition" is required. Indispensably, the technique must be applicable at elevated temperature in boiling conditions.

The ultrasonic velocity profiler (UVP) is an excellent technique for obtaining spatial-temporal velocity distribution in liquid flows [10]. With UVP, the ultrasonic transducer emits an ultrasonic pulse into the liquid and receives the echo signal backscattered from the reflector (particle) dispersed in the flow field. The velocity distribution along the sound path is computed by analyzing the echo signals. Since the ultrasonic signal can penetrate through various materials, optical access is not required. Hence, this method is a non-intrusive measurement that can work in an opaque fluid without a specific procedure and setup. Moreover, the negative effect on the measurement caused by the bubble overlapping problem is minimized because the measurement region of the UVP is in volume line form throughout the measurement depth. This technique has been applied for obtaining velocity profiles in several liquids such as water, liquid metal, magnetic liquid, and liquid sodium [11-14]. Furthermore, apart from the velocity profile measurement, the UVP can be utilized in several applications, such as liquid leakage investigation [15], measurement of the velocity structure and particle concentration profile [16], estimation of the maturity and inner pulp structures of fresh fruit [17], etc.

Regarding bubbly flow, Aritomi et al. [18] developed a hybrid system that integrates the UVP measurement and video data processing units to investigate the velocity distribution of liquids and bubbles. However, separation is limited when the velocity of both phases is not significantly different. Suzuki et al. [19] proposed the UVP method in conjunction with the phase-separation technique. This method applied the pattern recognition of the velocity level to distinguish a velocity profile phase. However, the technique could only be applied in limited flow conditions. Murakawa et al. [20] employed a multi-wave TDX transducer to measure instantaneous liquid and bubble velocity profiles. The pattern recognition of ultrasonic-echo amplitude was used to distinguish the velocity in both phases. However, the technique requires additional devices since two resonant frequencies are needed. Furthermore, sometimes the instantaneous full profile of the liquid velocity cannot be measured due to bubbles occurring simultaneously in the wave paths of both frequencies.

Wongsaroj et al. [21] represented the UVP with single ultrasonic gas-liquid two-phase separation (SUTS), which relies on combining time-frequency analysis and Doppler amplitude classification. The method can measure the instantaneous velocity profile of bubbles and liquid in bubbly flow using a single frequency, with measurement accuracy guaranteed by the excellent agreement in comparison to the PIV method. The discrepancy was $\pm 10 \%$. This technique can be utilized in air-water bubbly flow where the fluid is measured in low temperature, and the bubble made from pneumatic air. It has not yet been applied in vapor-liquid boiling conditions, which is the aim of this research. The UVP has an application opportunity. Therefore, the applicability of the UVP with the SUTS technique on vapor-liquid boiling bubbly flow must be proven.

This study presents the proposed application of the UVP integrated with the SUTS technique in vapor-liquid bubbly flow. The ultrasonic transmission in the water at ambient temperature until reaching boiling temperature is investigated. The Doppler amplitude threshold for separating the bubble and liquid data is set statistically, while the velocity profile measurement of the single-phase water at elevated temperature is preliminarily tested. The experimental measurement is then demonstrated in the vapor-liquid boiling bubbly flow, with the vapor bubble generated by a heating rod. The velocity profile of the vapor bubble and the liquid is derived experimentally. 


\section{Measurement Technique}

Fig. 1 shows the UVP configuration and velocity profile reconstruction. An ultrasonic pulse is transmitted from the transducer to the liquid throughout the measurement line. The echo signal reflected from the moving reflector (such as a tiny particle dispersed in the fluid) is obtained by the same transducer. When the ultrasonic pulse is emitted repeatedly, the echo signals are obtained sequentially. The Doppler signal influenced by the velocity of moving particles can be demodulated from the echo signals. The Doppler frequency $f_{\mathrm{D}}(i)$ estimated certainly relates to the particle's velocity ( $i$ is the position or channel). Hence, the velocity of the particle at that position $V(i)$ can be calculated as:

$$
V(i)=\frac{c f_{D}(i)}{2 f_{0} \sin \theta}
$$

where $c$ is the speed of sound in the fluid, $f_{0}$ means the basic frequency of the ultrasonic pulse, and $\theta$ is the incident angle. The particle closely follows the liquid streamline if the Stokes number of the liquid and small particle dispersed is lower than 0.1 . If there is a dispersion of several particles in the liquid, the velocity profile can be obtained.

The UVP with the SUTS technique was developed in the work of Wongsaroj et al. [21] to measure the velocity profile in air-water bubbly flow. It is the technique employed to measure the velocity distribution of vapor-liquid boiling bubbly flow in this study, based on the pulse repetition technique. Fig. 2 illustrates the process of the UVP with SUTS technique. Firstly, the Doppler signal $D(n)$ in discrete form, as shown in Eq. (2), is extracted from the echo signals obtained from the transducer and pulser/receiver, respectively. The extraction process is performed in the quadrature demodulation section [22].

$$
D_{i}(n)=A_{n, i} \cos \left(\frac{2 \pi n f_{D}}{f_{P R F}}-\varphi\right)-i A_{n, i} \sin \left(\frac{2 \pi n f_{D}}{f_{P R F}}-\varphi\right)
$$

where $A$ is the signal amplitude, $\varphi$ is the initial phase, and $n$ represents the sample Doppler signal.

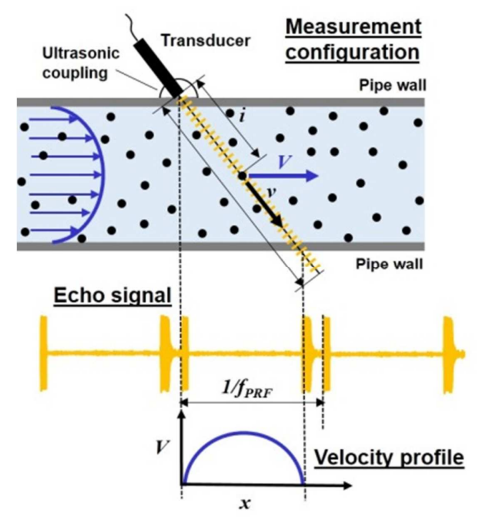

Fig. 1 UVP principle

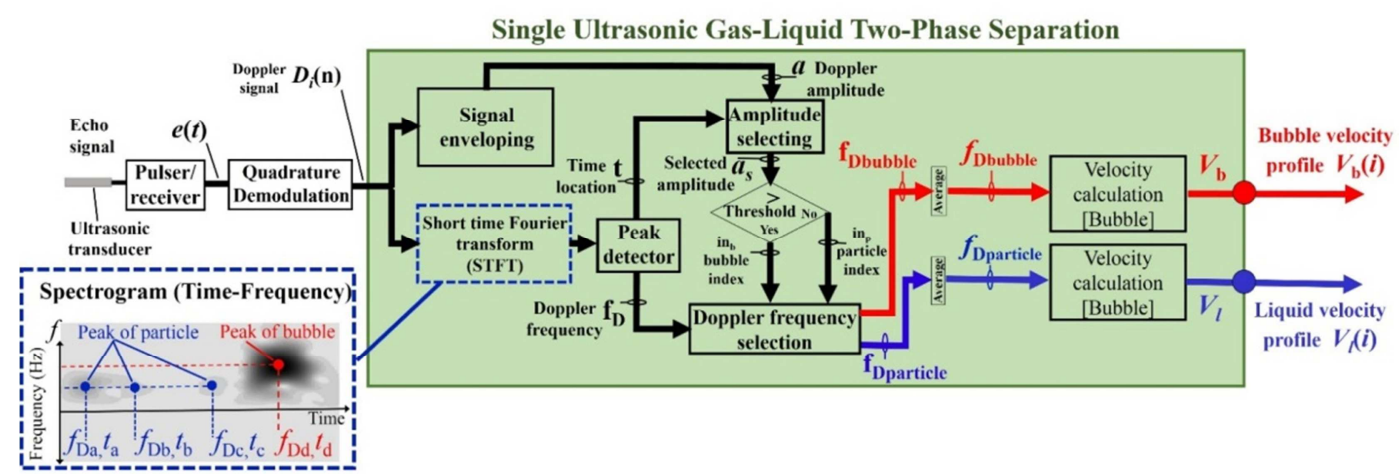

Fig. 2 Schematic of the UVP with the SUTS technique 
The Doppler signal is subsequently sent to the short-time Fourier transform (STFT) function and the time-frequency spectrogram of the signal computed. The calculation is expressed in Eq. (3), and the energy density of spectra at time $k$ is calculated by Eq. (4). The time-frequency resolution certainly alters following time step $S_{\mathrm{n}}$ and window length $W_{\mathrm{n}}$. The spectrogram is sent to the peak detector to analyze the energy peaks. The peak value in each position informs the Doppler frequency data $\left(f_{\mathrm{D}}=f_{\mathrm{Da}}, f_{\mathrm{Db}}, \ldots, f_{\mathrm{Dm}}\right)$ and time location $\left(t=t_{\mathrm{a}}, t_{\mathrm{b}}, \ldots, t_{\mathrm{m}}\right)$. Furthermore, the Doppler amplitude in each point $(a=$ $\left.a_{\mathrm{n}=0}, a_{\mathrm{n}=1}, \ldots, a_{\mathrm{n}=\mathrm{Nrep}-1}\right)$ is detected by envelope processing the Doppler signal. The data is then selected according to the time location index obtained from the peak detector $\left(a_{\mathrm{S}}=a_{\mathrm{a}}, a_{\mathrm{b}}, \ldots, a_{\mathrm{m}}\right)$.

$$
\begin{aligned}
& X\left(k, f_{D}\right)=\sum_{n=0}^{N_{R E P}-1} D(n) W(n-k S) \exp \left(-j n 2 \pi f_{D}\right) \\
& P\left(k, f_{D}\right)=\left|X\left(k, f_{D}\right)\right|^{2}
\end{aligned}
$$

The selected amplitude data is derived and compared with the threshold value which is set to be higher than the maximum Doppler amplitude of the particle. Hence, the bubble and liquid index is classified by this comparison. If the amplitude value is higher than the threshold, this is defined as a bubble index $\left(i n_{\mathrm{b}}=i n_{\mathrm{b} 1}, i n_{\mathrm{b} 2}, \ldots, i n_{\mathrm{bn}}\right)$. Furthermore, when the value is lower than the threshold, this is expressed as a particle index $\left(i n_{\mathrm{p}}=i n_{\mathrm{p} 1}, i n_{\mathrm{p} 2}, \ldots, i n_{\mathrm{pn}}\right)$. The Doppler frequency data analyzed by the peak detector is distinguished as the Doppler frequency of the bubble group $\left(f_{\text {Dbubble }}=f_{\mathrm{Dba}}, f_{\mathrm{Dbb}}, \ldots, f_{\mathrm{Dbm}}\right)$ and particle group $\left(f_{\mathrm{Dparticle}}=\right.$ $\left.f_{\mathrm{Dpa}}, f_{\mathrm{Dpb}}, \ldots, f_{\mathrm{Dpm}}\right)$ using these amplitude indexes, and the Doppler frequencies in each group subsequently averaged. Hence, the Doppler frequency of bubble $f_{\text {Dbubble }}$ and particle $f_{\text {Dparticle }}$ in the same measurement channel is decomposed. Consequently, by placing the Doppler frequencies according to Eq. (1), bubble and particle (liquid tracer) velocity can be calculated simultaneously. The reflected echo signal consists of several measurement volumes or channels over the sound beam. Hence, the velocity profile of bubbles and liquid can be obtained simultaneously.

\section{Experimental Setup}

The UVP with the SUTS technique must be verified experimentally to measure the velocity profile of the liquid flow at elevated temperature and vapor-liquid boiling bubbly flow, respectively. The vertical pipe flow apparatus illustrated in Fig. 3 is utilized for the experiment. The test section is located on a vertical polycarbonate pipe with an inner diameter $(D)$ of $50 \mathrm{~mm}$. Polycarbonate can effectively withstand the fluid temperature in boiling conditions since its melting and mold points are higher than acrylic. Tap water dispersed with $80 \mu \mathrm{m}$ nylon particles works as a liquid phase and the pump activates the water circulation from the bottom tank to the top. The water flow rate is measured by the orifice flow meter, which can withstand high-temperature fluid. The vapor bubble is produced by inserting a heating rod into the main pipe upstream of the test section with the end located $6 \mathrm{D}$ from it. The water is preheated by the heating coil immersed in the bottom tank while the enhanced temperature produced by the heater is regulated by the controller.

The fluid temperature is measured by a thermocouple at three measurement locations: the test section (the location does not disturb the wave path of the transducer), the upstream of the heating rod, and the bottom tank. The temperature data from the bottom tank is used to control the preheating of the fluid in the bottom tank. The ultrasonic transducers at a basic frequency of $4 \mathrm{MHz}$ are installed by submerging them into the water box in the test section. The water in the water box (test section) is circulated to cool down its temperature and protect the transducer from damage due to the elevated temperature $\left(\geq 60{ }^{\circ} \mathrm{C}\right)$ resulting from the influence of thermal conduction in the boiling flow inside the main pipe. Table 1 represents the specification of the UVP measurement system used in this experiment. The UVP parameter setting is shown in Table 2. 


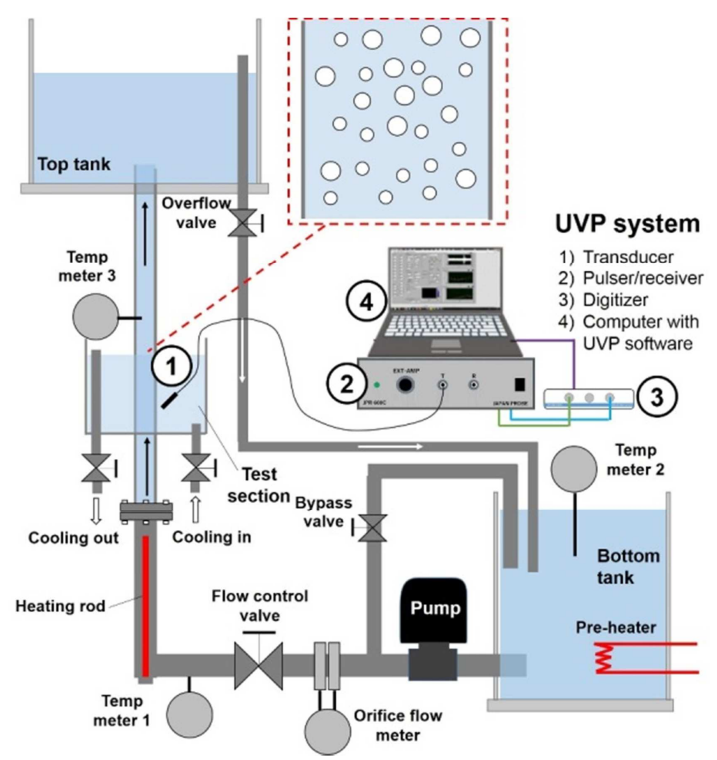

Fig. 3 Experimental apparatus

Table 1 Specification of the UVP system

\begin{tabular}{|c|c|}
\hline Name & Detail \\
\hline Transducer & 4 MHz, Model: TX-4-5-8, MFG: Met-Flow \\
\hline Pulser/receiver & Model: JPR 600C, MFG: Japan probe \\
\hline Digitizer & Model: NI USB 5133, MFG: National Instrument \\
\hline Computer & Model: Vostro, MFG: Dell \\
\hline
\end{tabular}

Table 2 UVP parameter setting

\begin{tabular}{|c|c|}
\hline Parameter & Value \\
\hline Basic frequency $\left(f_{0}\right)$ & $4 \mathrm{MHz}$ \\
\hline Emission voltage and gain & $150 \mathrm{~V}_{\mathrm{p}-\mathrm{p}}, 45 \mathrm{~dB}$ \\
\hline Pulse repetition frequency $\left(f_{\mathrm{PRF}}\right)$ & $4 \mathrm{kHz}$ \\
\hline Number of repetitions $\left(N_{\mathrm{REP}}\right)$ & 128 \\
\hline Number of cycles per pulse & 4 \\
\hline Ultrasonic-echo sampling frequency & $100 \mathrm{MS} / \mathrm{s}$ \\
\hline Channel width $(w)$ & $0.74 \mathrm{~mm}$ \\
\hline
\end{tabular}

\section{Preliminary Testing}

\subsection{Ultrasound transmission at elevated temperature}

The aim of the proposed UVP with the SUTS technique is to measure the velocity distribution in boiling conditions at ambient pressure. Its temperature is $\approx 90{ }^{\circ} \mathrm{C}$ to $100{ }^{\circ} \mathrm{C}$. The normal transducer cannot contact the fluid directly at this temperature level due to its maximum allowable temperature being $60^{\circ} \mathrm{C}$. The internal components of the disbanding transducer will cause damage if employed beyond this limit, and therefore, the ultrasonic coupling with cooling conditions must be applied. This experiment is conducted on the vertical pipe flow apparatus, and the cooling water is used as ultrasonic coupling. The temperature between the cooling water and boiling flow within the pipe is significantly different. Therefore, it is imperative to investigate the ability of the ultrasound transmission penetrating into the cooling water and fluid in the pipe. Fig. 4 shows the testing schematic. The convection of fluid temperature within the pipe distributed into the cooling water (ultrasonic coupling) risks damaging the transducer if it cannot cool down the accumulated temperature. The efficiency of the cooling water must be verified. The fluid temperature in the pipe (T1) and the cooling water temperature (T2) are measured and compared. Fig. 5 presents the results. The cooling water temperature could be kept at a low level ( $\approx$ ambient temperature) even if the fluid temperature within the pipe elevates to $95^{\circ} \mathrm{C}$. 
The ability of ultrasonic transmission into the water at boiling temperature is then investigated. The transducer emits an ultrasonic wave into the fluid in the pipe. The reflection from the far wall of the inner pipe is received by the same transducer and the fluid temperature in the pipe increases from $20^{\circ} \mathrm{C}, 50^{\circ} \mathrm{C}$, to $95^{\circ} \mathrm{C}$. The time-of-flight measurement at each temperature level is conducted and converted into sound velocity. The changing ratio $\left(c_{\mathrm{t}} / c_{20^{\circ} \mathrm{C}}\right)$ of the sound velocity is used to evaluate the ultrasonic transmission under the altering fluid temperature in the pipe, as illustrated in Fig. 6. The experimental result is in good agreement with the calculation.

Therefore, it can be concluded that the cooling water can protect against damage to the transducer caused by the high temperature coming from the boiling flow in the pipe. Furthermore, the ultrasonic wave can be transmitted into the fluid in the pipe even at boiling temperature. Its behavior is verified to agree with the theory. However, to measure the velocity profile using the UVP, the ultrasonic transducer must be installed at an inclined angle. When the transducer is installed in the test section, an ultrasonic wave penetrates the ultrasonic coupling (cooling water), pipe wall, and fluid in the pipe, respectively. The transformation mode of the ultrasonic wave occurs because of the refraction at the interface between mediums, as shown in Fig. 7.

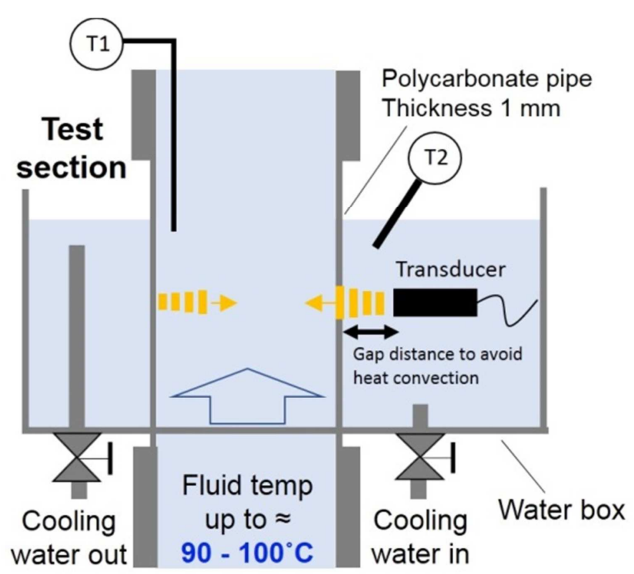

Fig. 4 Experimental setup for ultrasonic transmission testing

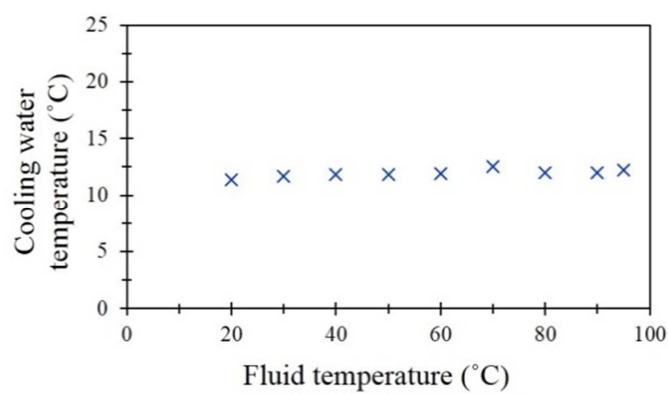

Fig. 5 Temperature of cooling water with the fluid temperature altering in the pipe

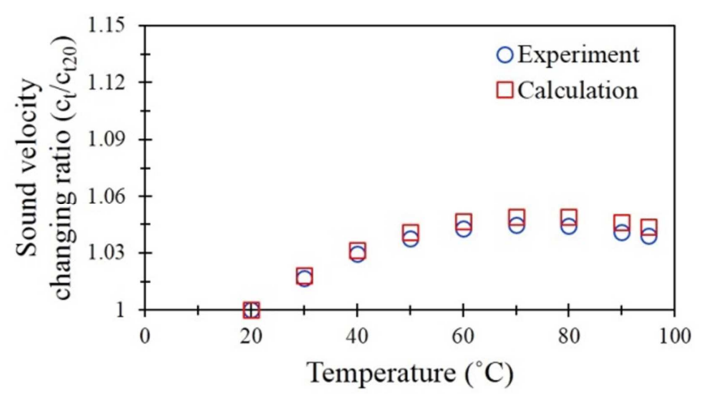

Fig. 6 Sound velocity changing ratio $\left(c_{\mathrm{t}} / c_{20^{\circ} \mathrm{C}}\right)$

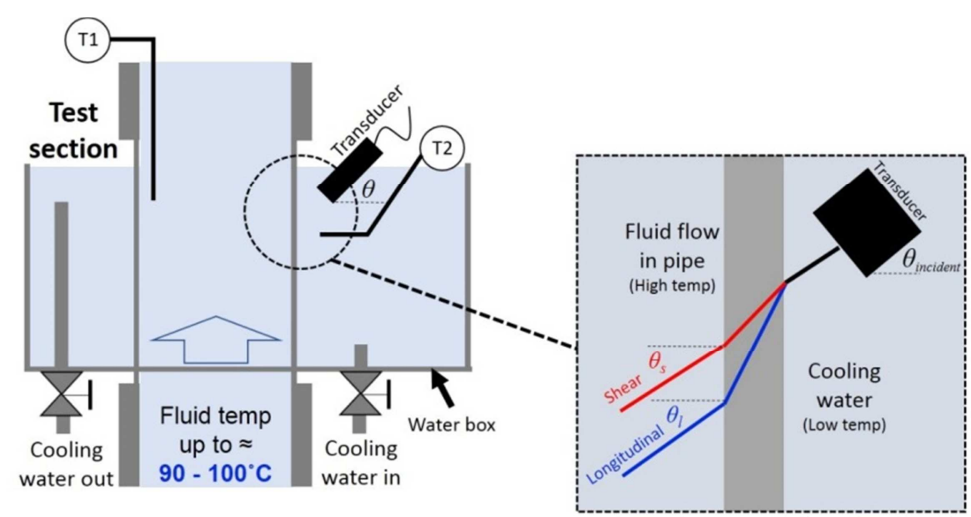

Fig. 7 Transformation mode in the pipe wall 


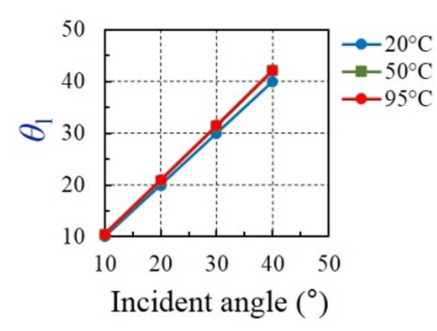

(a) Longitudinal path

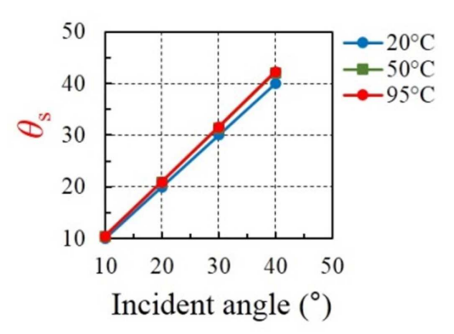

(b) Shear path

Fig. 8 Relationship between the incident angle and wave path angle when the fluid in the pipe is at an elevated temperature

To accurately measure the velocity profile, the wave path angle in the fluid and the incident angle must be confirmed. Fig. 8 represents the calculation result of the relationship between the incident angle and the wave path angle of the fluid in the pipe (longitudinal and shear path) under a changing incident angle. The result shows that altering the difference between the temperature of cooling water and fluid in the pipe influences the mismatch of these angles, especially at a high-temperature difference. Therefore, it is compulsory that the fluid temperature in the pipe is measured and used to compensate for the velocity measurement to reduce the error emanating from this effect.

\subsection{Doppler amplitude threshold}

In order to separate the vapor bubble and liquid data, the Doppler amplitude threshold must be set. In this section, the water flow dispersed by the $80 \mathrm{~mm}$ nylon particles (tracer particles) is set at a velocity of $U_{\mathrm{L}} 100 \mathrm{~mm} / \mathrm{s}$, and the Doppler amplitudes are then observed respectively. For application in the vapor-liquid boiling bubbly flow with elevated fluid temperature, the Doppler amplitude must be investigated to ascertain the effects. Hence, it is observed at various bulk liquid temperatures $t_{\mathrm{b}}$, i.e., $20^{\circ} \mathrm{C}, 50^{\circ} \mathrm{C}$, and $95^{\circ} \mathrm{C}$, respectively (the temperature measurement point is located around the test section).

Firstly, the incident angle is set at $45^{\circ}$, as shown in Fig. 9(a). This angle provides good sensitivity and a single wave path for evaluating the acrylic pipe [23]. There are 96 measurement channels (channel distance: $0.74 \mathrm{~mm}$ ) within the $50 \mathrm{~mm}$ inner diameter of the pipe. In this experiment, the heating coil installed in the bottom tank is turned on to preheat the water. The heating rod inserted in the main pipe is deactivated (the vapor bubble is not generated). A total of 288,000 Doppler signals are recorded in each temperature level obtained by the receiver according to the measurement channels within the inner pipe, and the maximum amplitude values categorized in each channel. The data is reformed to become the probability density function (PDF) and then categorized in each measurement channel and plotted on the spectrogram as shown in Figs. 10(a)-(c). The probability of the Doppler amplitude data of particles occurring at the point $=0.99$ is then plotted. The Doppler amplitudes obtained at this incident angle in each temperature level are very small. Fig. 10(d) shows the echo signal at $95^{\circ} \mathrm{C}$ and also illustrates that it has a small amplitude in the period of reflection from the moving particle in the pipe.

Fig. 11(a) represents the water velocity profile computed in each temperature level by utilizing the derived Doppler amplitudes. Also, the sound velocity in the water is set at an appropriate level for each water temperature. The average velocity data is calculated from 3,000 items of instantaneous data. The velocity profile on the measurement line can be plotted. The horizontal axis refers to the inner pipe distance. The profiles derived are inaccurate, with the profile at the near-wall and far-wall region being asymmetric. The standard deviation of the averaged profiles is very high, as shown in Fig. 11(b), especially at a liquid temperature of $95^{\circ} \mathrm{C}$. The problem is caused by inefficient penetration of ultrasonic transmission into the polycarbonate pipe and measuring fluid, respectively, and vice versa [24]. The refractions of an ultrasonic wave in these interfaces significantly influence the transformation mode occurring inside the pipe, i.e., longitudinal and shear path. At the interface between the pipe and the measuring liquid, both wave paths in the pipe are subsequently transformed into only longitudinal waves to penetrate the liquid. Both paths occur in different locations. 


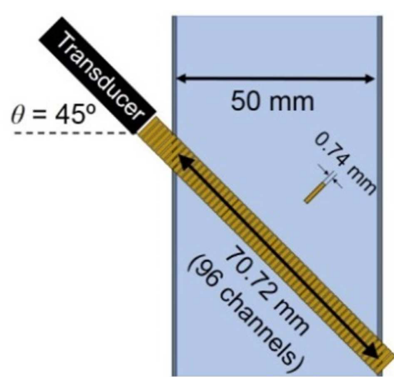

(a) $\theta=45^{\circ}$

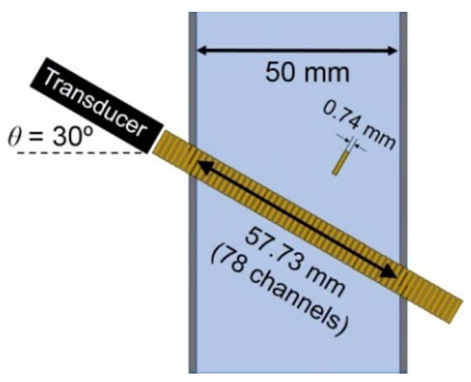

(b) $\theta=30^{\circ}$

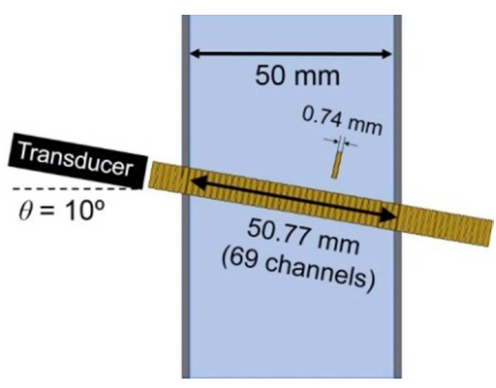

(c) $\theta=10^{\circ}$

Fig. 9 Transducer installation and measurement length

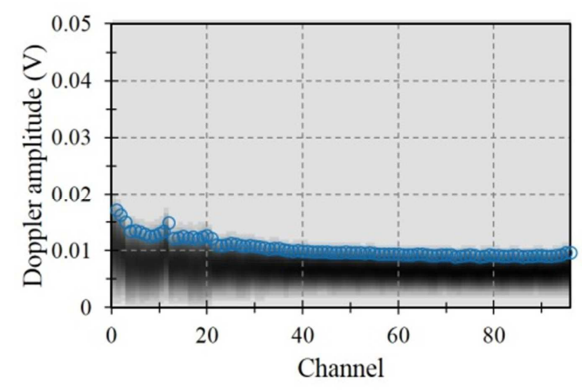

(a) Doppler amplitude at $20^{\circ} \mathrm{C}$

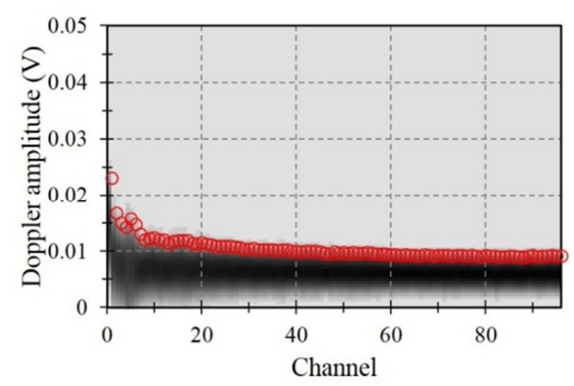

(c) Doppler amplitude at $95^{\circ} \mathrm{C}$

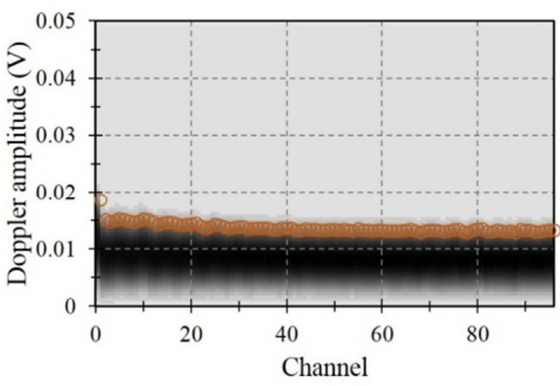

(b) Doppler amplitude at $50^{\circ} \mathrm{C}$

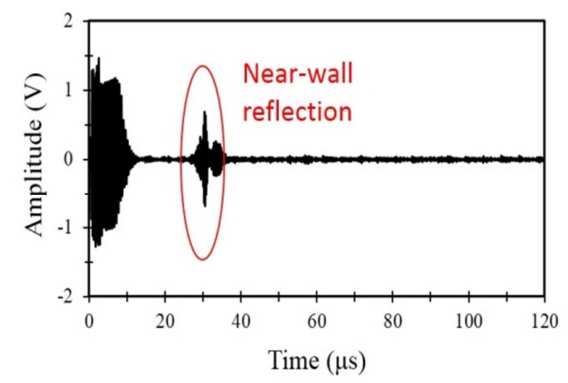

(d) Echo signal at $95^{\circ} \mathrm{C}$

Fig. 10 PDF of the maximum Doppler amplitude and echo signal at a $45^{\circ}$ incident angle

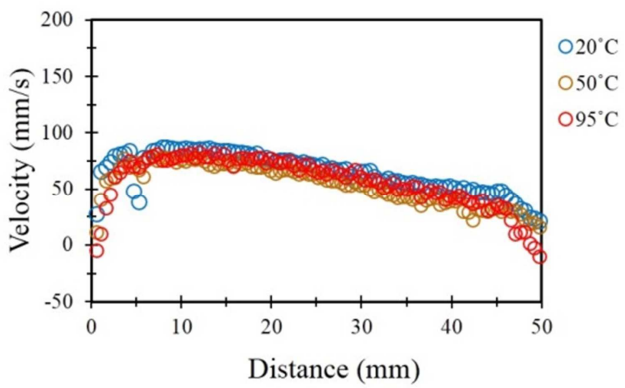

(a) Velocity profile

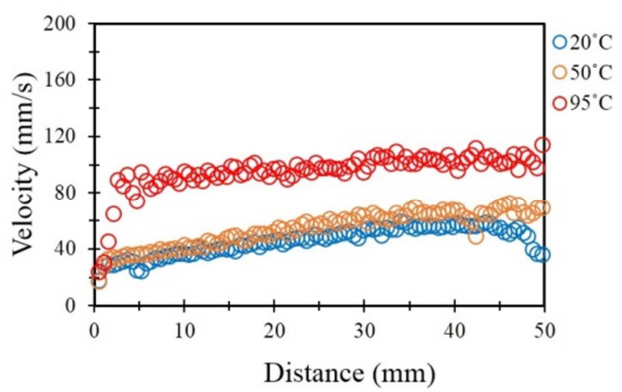

(b) Standard deviation

Fig. 11 Average liquid velocity and standard deviation with an incident angle of $45^{\circ}$

Fig. 12 illustrates the ultrasonic transmission efficiency of the acrylic and polycarbonate pipe [24]. The highest transmission efficiency in the acrylic pipe is indicated at an incident angle of $45^{\circ}$ on the shear wave path. On the contrary, in the polycarbonate pipe, the worst ultrasonic transmission occurs between the incident angles of $40^{\circ}$ and $45^{\circ}$. This region is called the critical angle. The incident angle makes the angle of refraction for the longitudinal and shear wave reach $90^{\circ}$. For longitudinal waves, all the energy from the refracted longitudinal wave is converted into a surface at the first critical angle. The incident angle makes the angle of refraction for the shear wave reach $90^{\circ}$. This is the second critical angle, and at this point, all the wave energy is refracted into a surface. The critical angles on the pipe wall of both materials are different due to the variation in sound velocity. Therefore, for the polycarbonate pipe, other incident angles are considered. The angle must be smaller than $45^{\circ}$ to avoid measurement error from a large angle. Angles of $30^{\circ}$ and $10^{\circ}$ are therefore selected. 


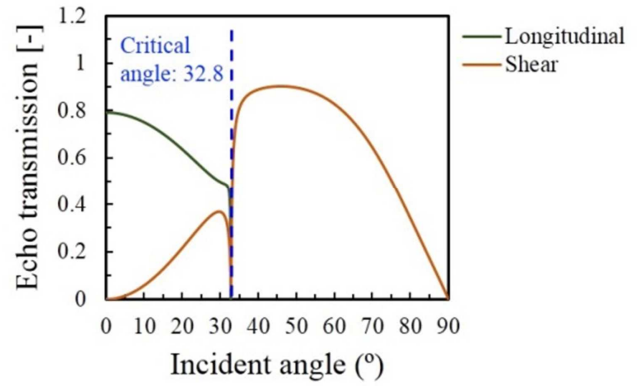

(a) Acrylic

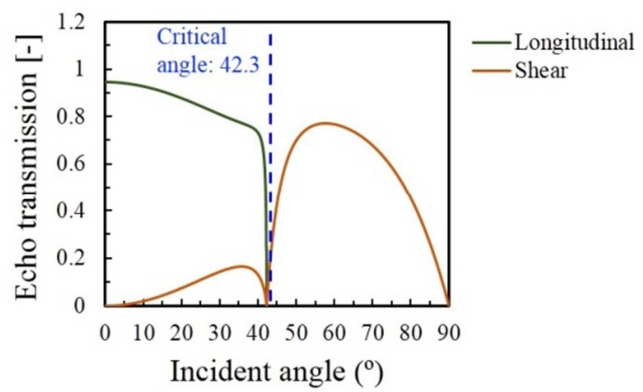

(b) Polycarbonate

Fig. 12 Ultrasonic transmission efficiency

At an incident angle of $30^{\circ}$, there are 78 measurement channels within the inner pipe, as shown in Fig. 9(b). The Doppler amplitude obtained in all measurement channels for different fluid temperatures is bigger than the previous incident angle, as represented in Figs. 13(a)-(c). The echo signal obtained is reasonably large according to the Doppler signal collected, as shown in Fig. 13(d). Fig. 14(a) shows the mean of 3,000 instantaneous velocity profiles computed by utilizing the collected Doppler signal at this incident angle, indicating an accurate measurement result. The profiles at the near-wall and far-wall region are symmetrical. The standard deviation of the averaging profiles is smaller than the previous incident angle, as shown in Fig. 14(b).

Besides, at an incident angle of $10^{\circ}$, there are 69 measurement channels within the inner pipe, as shown in Fig. 9(c). The Doppler amplitude derived in all measurement channels at a fluid temperature of $95^{\circ}$ is similar in size to the amplitude at an incident angle of $30^{\circ}$, as shown in Fig. 15(a). The echo signal obtained at this angle is shown in Fig. 15(b). However, the velocity profile computed is very ambiguous, as represented in Fig. 16(a), with a high standard deviation being observed. The problem is caused mainly by a very small incident angle, as illustrated in Fig. 16(b). Hence, it can be concluded that the selected incident angle is $30^{\circ}$. The Doppler amplitude thresholds are set at an incident angle of $30^{\circ}$ for the elevated fluid temperature. The amplitude data of the particle is plotted as a PDF, in which the probability $=0.99$ (particle zone) in each fluid temperature must be evaluated. Fig. 17(a) shows the comparable data in each water temperature. The similarity in Doppler amplitude data of each fluid temperature for all measurement channels can be clearly observed. Fig. 17(b) represents the equation of the Doppler amplitude threshold based on the polynomial function. This equation is employed to separate the velocity of vapor bubble and liquid in boiling bubbly flow in the next section.

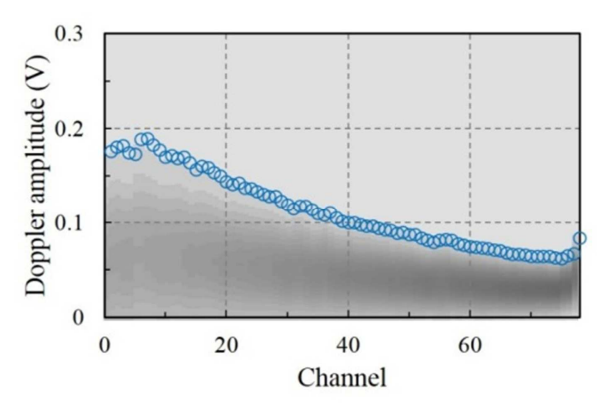

(a) Doppler amplitude at $20^{\circ} \mathrm{C}$

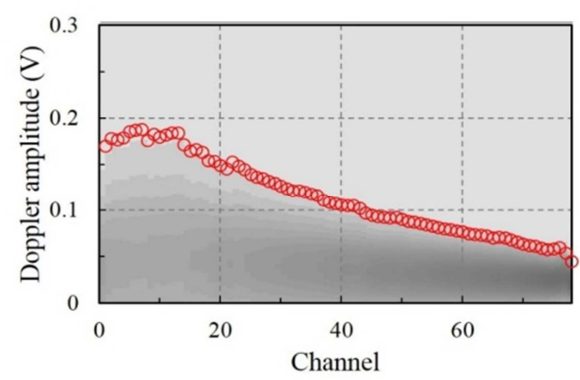

(c) Doppler amplitude at $95^{\circ} \mathrm{C}$

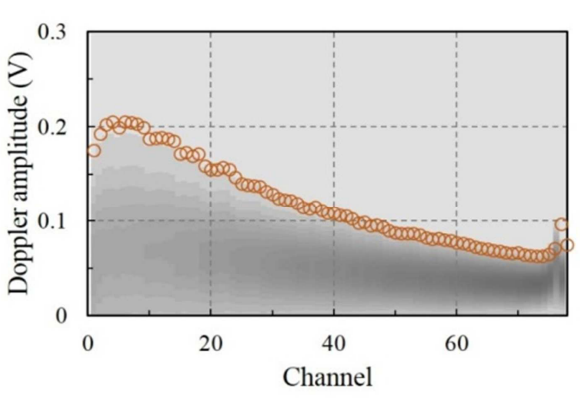

(b) Doppler amplitude at $50^{\circ} \mathrm{C}$

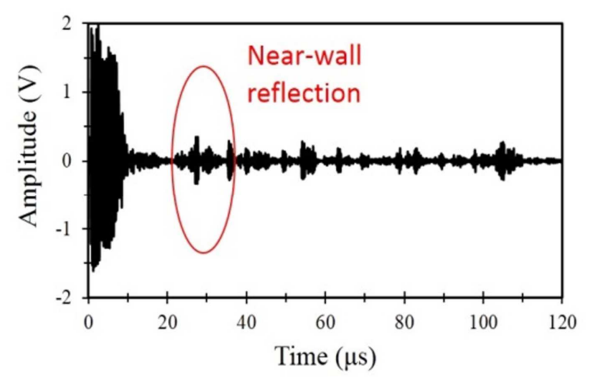

(d) Echo signal at $95^{\circ} \mathrm{C}$

Fig. 13 PDF of the maximum Doppler amplitude and echo signal at incident angle of $30^{\circ}$ 


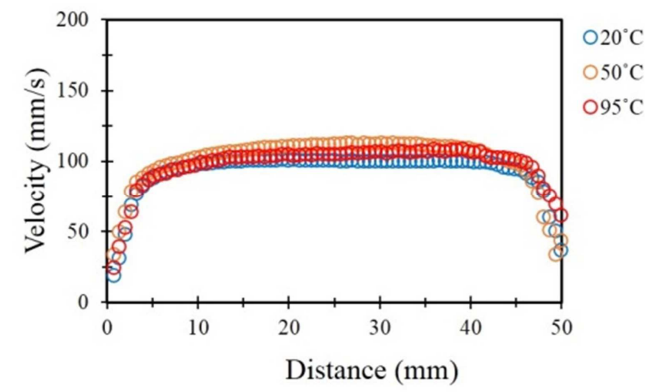

(a) Velocity profile

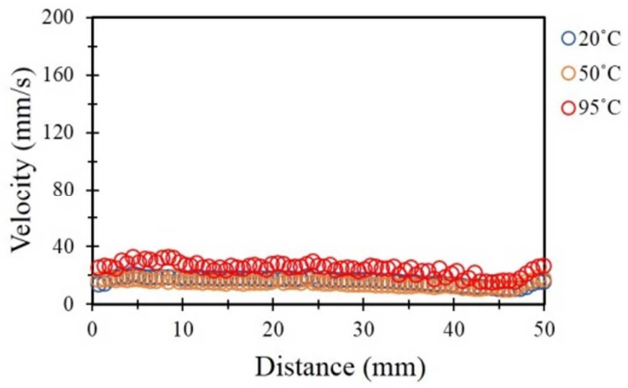

(b) Standard deviation

Fig. 14 Average liquid velocity with an incident angle of $30^{\circ}$

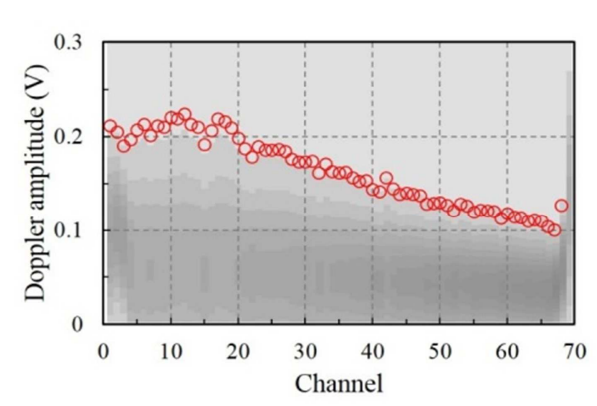

(a) PDF of Doppler amplitude

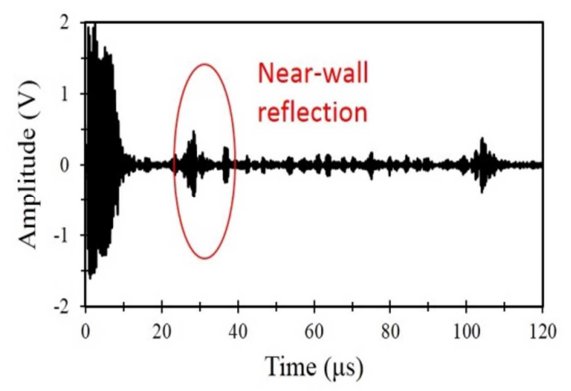

(b) Echo signal

Fig. 15 PDF of the maximum Doppler amplitude and echo signal at an incident angle of $10^{\circ}$

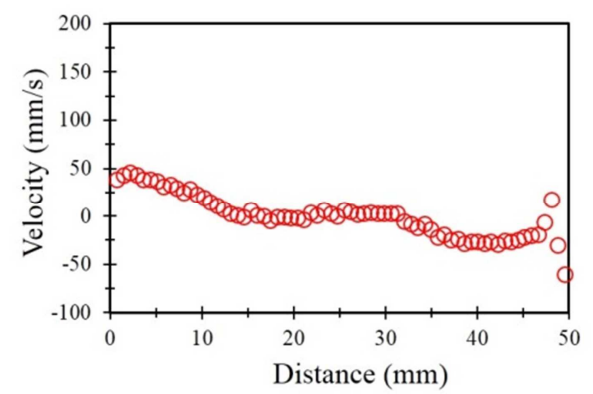

(a) Velocity profile

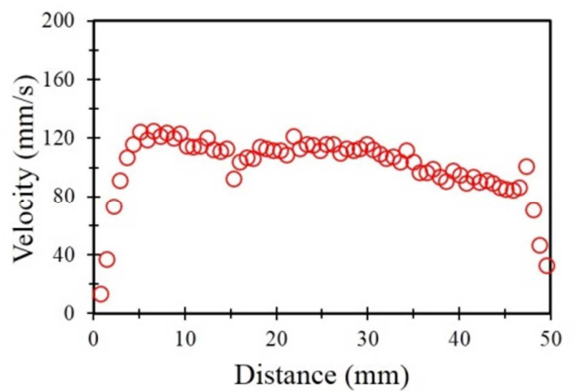

(b) Standard deviation

Fig. 16 Average liquid velocity with an incident angle of $10^{\circ}$

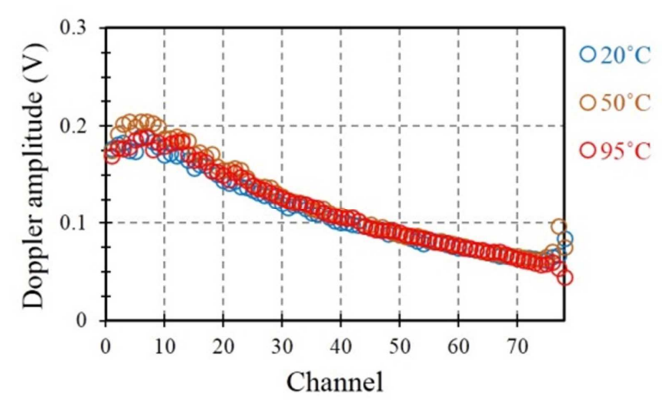

(a) Doppler amplitude at $20^{\circ} \mathrm{C}, 50^{\circ} \mathrm{C}$, and $95^{\circ} \mathrm{C}$

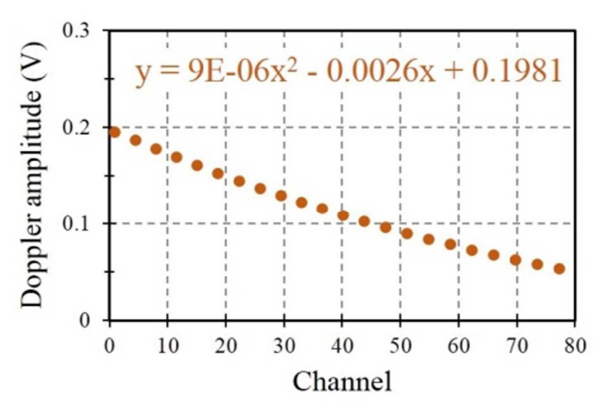

(b) Doppler amplitude equation

Fig. 17 The maximum Doppler amplitude ( 0.99 probability) at incident angle $30^{\circ}$ in different fluid temperature

\section{Measurement Results and Discussion}

In this section, an experiment is conducted to obtain the velocity profile of the vapor bubble and liquid in boiling conditions using the UVP with the SUTS technique. The bulk liquid temperature $t_{\mathrm{b}}$ is set at $95^{\circ} \mathrm{C}$ and the heating coil is energized to preheat the water in the bottom tank. The heating rod is powered up to generate the vapor bubble at a small flow rate. The $U_{\mathrm{L}}$ is set at $\approx 20$ $\mathrm{mm} / \mathrm{s}$ for effective heating accumulation due to the limitation of the heater employed in the experiment. The Doppler amplitude thresholds of $95^{\circ} \mathrm{C}$ proposed in the previous section are applied, and the speed of sound is set at $1550 \mathrm{~m} / \mathrm{s}$. 
Firstly, the rod is heated at low power (800 W). Fig. 18(a) shows a real image of the bubbly flow during the experiment when the vapor bubble occurs. The diameters of the bubbles are mostly smaller than $2 \mathrm{~mm}$, as shown in Fig. 18(b). The image processing technique is used to determine the bubble diameter distribution. The technique utilizes a high-speed camera with a backlight to capture the bubble image and area to calculate the equivalent bubble diameter. Fig. 19 illustrates the results of the bubbly flow velocity profile, according to the average data from 3,000 instantaneous profiles. The velocity profile of both phases can be obtained separately. The velocity level of the vapor bubble is about $80 \mathrm{~mm} / \mathrm{s}$ at the central region of the inner pipe and $50 \mathrm{~mm} / \mathrm{s}$ at the wall zone. The effect of rising bubbles increases the velocity level of the liquid phase. The liquid velocity is approximately $60 \mathrm{~mm} / \mathrm{s}$ in the central zone. In this experiment, the vapor bubble generated is small, influencing a low buoyancy force. Hence, a small slip ratio in the velocity of both phases could be seen in this case.

Next, to generate a bigger vapor bubble, substantial heat needs to be accumulated around the heating rod, so the heating power is increased to $1200 \mathrm{~W}$. The $t_{\mathrm{b}}$ is still controlled at $95^{\circ} \mathrm{C}$ and the $U_{\mathrm{L}}$ is set at $\approx 20 \mathrm{~mm} / \mathrm{s}$. The amplitude thresholds and sound velocity are similar to those set in the previous experiment. Fig. 20(a) shows an actual image of the bubbly flow during this experiment. The bubble size distribution is bigger than the previous case, as shown in Fig. 20(b). Fig. 21 represents the average velocity profile of the bubbly flow calculated from 3,000 instantaneous profiles. The bubble size is larger than the previous case to enhance the buoyancy force. The velocity level of the bubble increases to $150 \mathrm{~mm} / \mathrm{s}$ at the central zone within the inner pipe-the velocity level at the wall zone is about $30 \mathrm{~mm} / \mathrm{s}$ lower than the value at the central region. As a consequence of the bubble rising due to its larger size, the liquid velocity increases to $100 \mathrm{~mm} / \mathrm{s}$ at the central region of the pipe. The slip ratio, in this case, could obviously be observed as being significantly larger than the previous case.

The measurement result obtained by the UVP in both experiments is validated by PIV as represented in Figs. 19 and 21. Due to the high number of bubbles, the light source projected by the laser sheet makes it impossible to penetrate fluid effectively. The PIV camera cannot capture the image of the bubble and tracer particle motion. Hence, in this experiment, the PIV with the backlight is applied to derive only the bubble velocity profile. Although the bubble velocity profile result obtained from both techniques is not precisely in good agreement, both results have a similar trend, and there is a corresponding change in the result according to the altering fluid velocity. It can be summarized that the UVP with the SUTS technique is applicable for measuring the velocity profile of vapor-liquid bubbly flow for experimental investigation.

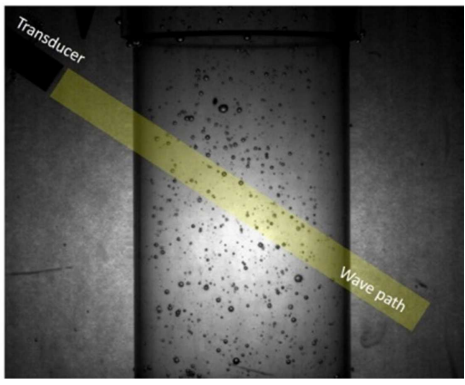

(a) Image of bubbly flow

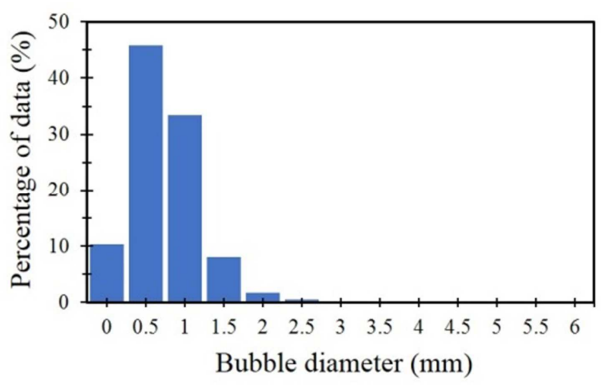

(b) Bubble diameter distribution

Fig. 18 Image and bubble diameter distribution of vapor-liquid bubbly flow at low heat $(800 \mathrm{~W})$

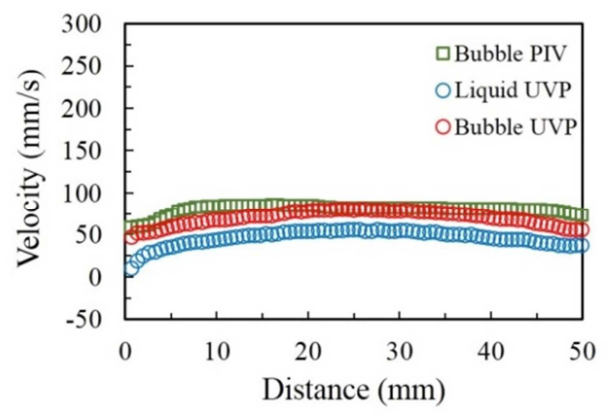

Fig. 19 Average velocity profile of vapor-liquid bubbly flow at low heat $(800 \mathrm{~W})$ 


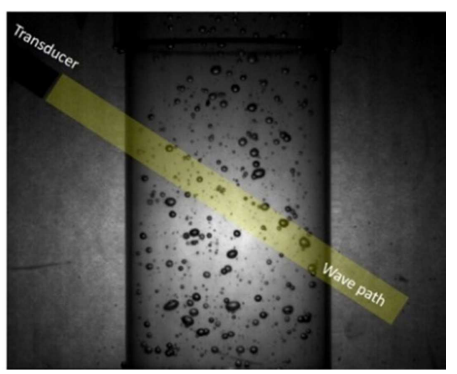

(a) Image of bubbly flow

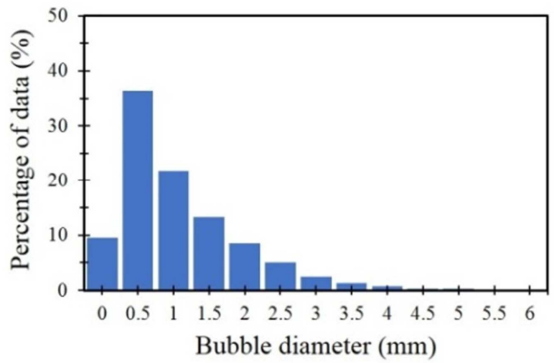

(b) Bubble diameter distribution

Fig. 20 Image and bubble diameter distribution of vapor-liquid bubbly flow at low heat (1200 W)

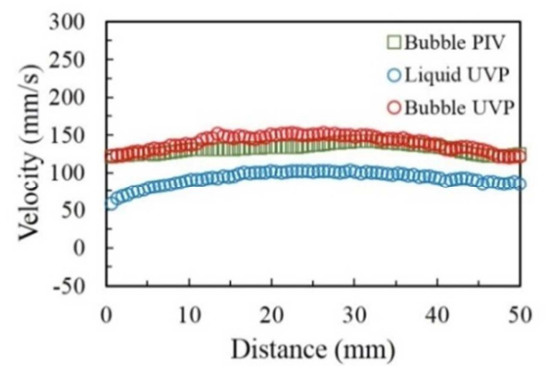

Fig. 21 Average velocity profile of vapor-liquid bubbly flow at low heat (1200 W)

\section{Conclusions}

The UVP with the SUTS technique is proposed in this study to measure the velocity distribution of vapor-liquid bubbly flow. The velocity profile of bubbles and liquid can be obtained separately. The SUTS represents the integration of time-frequency analysis and Doppler amplitude classification. The experiment conducted on the vertical pipe flow apparatus is designed to verify the ability of the UVP with the SUTS technique in the proposed application. The important experimental findings and results are summarized below.

(1) The ultrasonic transducer can be applied in the proposed technique since the circulated cooling water for ultrasonic coupling is utilized to cool down the high temperature around the transducer.

(2) The ultrasonic transmittance of the employed transducer can penetrate the cooling water and high-temperature water within the pipe, reflecting the applicability of the proposed technique.

(3) The vapor and liquid phase velocity data are separated by applying the Doppler amplitude threshold set under the statistical strategy.

(4) The measurement demonstrated in the vapor-liquid bubbly flow proves the performance of the UVP with the SUTS technique and the generation of a vapor bubble by a heating rod. The velocity profile in the vapor-liquid bubbly flow is derived separately.

(5) This study shows the effectiveness of the proposed measurement technique in experimental investigation to elucidate the complicated two-phase flow behavior in the boiling region for high performance and safety in a heat exchange system.

\section{Conflicts of Interest}

The authors declare no conflict of interest.

\section{References}

[1] G. E. Thorncroft, J. F. Klausner, and R. Mei, “An Experimental Investigation of Bubble Growth and Detachment in Vertical Upflow and Downflow Boiling,” International Journal of Heat and Mass Transfer, vol. 41, no. 23, pp. 3857-3871, December 1998. 
[2] G. E. Thorncroft and J. F. Klausner, "The Influence of Vapor Bubble Sliding on Forced Convection Boiling Heat Transfer," Journal of Heat Transfer, vol. 121, no. 23, pp. 73-79, February 1999.

[3] M. Ishii, Thermo-Fluid Dynamic Theory and Two-Phase Flow, Paris: Eyerolles, 1975.

[4] G. Kocamustafaogullari and M. Ishii, "Foundation of the Interfacial Area Transport Equation and Its Closure Relations," International Journal of Heat Mass Transfer, vol. 38, no. 3, pp. 481-493, February 1995.

[5] J. Yoo, C. E. Estrada-Perez, and Y. A. Hassan, "Experimental Study on Bubble Dynamics and Wall Heat Transfer Arising from a Single Nucleation Site at Subcooled Flow Boiling Conditions-Part 2: Data Analysis on Sliding Bubble Characteristics and Associated Wall Heat Transfer," International Journal of Multiphase Flow, vol. 84, pp. 292-314, September 2016.

[6] H. Anglart, "Modelling of the Multidimensional Phase Distribution in a BWR Fuel Assembly," Technical Meeting on Use of Computational Fluid Dynamics Codes for Safety Analysis of Reactor Systems, Including Containment, pp. 413-424, November 2002.

[7] J. L. Muñoz-Cobo, S. Chiva, S. Méndez, G. Monrós, A. Escrivá, and J. L. Cuadros, "Development of Conductivity Sensors for Multi-Phase Flow Local Measurements at the Polytechnic University of Valencia (UPV) and University Jaume I of Castellon (UJI)," Sensors, vol. 17, no. 5, 1077, May 2017.

[8] R. Lindken and W. Merzkirch, "Velocity Measurements of Liquid and Gaseous Phase for a System of Bubbles Rising in Water," Experiments in Fluids, vol. 29, no. 1, pp. 194-201, December 2000.

[9] A. Seeger, K. Affeld, L. Goubergrits, U. Kertzscher, and E. Wellnhofer, "X-Ray Based Assessment of the Three-Dimensional Velocity of the Liquid Phase in a Bubble Column,” Experiments in Fluids, vol. 32, no. 2, pp. 193-201, August 2001.

[10] Y. Takeda, "Velocity Profile Measurement by Ultrasonic Doppler Shift Method," International Journal of Heat and Fluid Flow, vol. 7, no. 4, pp. 313-318, December 1986.

[11] Y. Takeda, "Development of an Ultrasound Velocity Profile Monitor," Nuclear Engineering and Design, vol. 126, no. 2 pp. 277-284, April 1991.

[12] K. Kikuchi, Y. Takeda, H. Obayashi, M. Tezuka, and H. Sato, "Measurement of LBE Flow Velocity Profile by UDVP," Journal of Nuclear Materials, vol. 356, no. 1-3, pp. 273-279, September 2006.

[13] H. Kikura, Y. Takeda, and F. Durst, "Velocity Profile Measurement of the Taylor Vortex Flow of a Magnetic Fluid Using the Ultrasonic Doppler Method," Experiments in Fluids, vol. 26, no. 3, pp. 208-214, February 1999.

[14] S. Eckert and G. Gerbeth, "Velocity Measurements in Liquid Sodium by Means of Ultrasound Doppler Velocimetry," Experiments in Fluids, vol. 32, no. 5, pp. 542-546, May 2002.

[15] T. Moriya, H. Kikura, and H. Takahashi, "Basic Study on Ultrasonic Remote Leakage Position Estimation Method for Underwater Exploration,” 13th International Symposium on Ultrasonic Doppler Methods for Fluid Mechanics and Fluid Engineering, pp. 1-4, June 2021.

[16] J. Hitomi, S. Nomura, Y. Murai, G. De Cesare, Y. Tasaka, Y. Takeda, et al., "Measurement of the Inner Structure of Turbidity Currents by Ultrasound Velocity Profiling," International Journal of Multiphase Flow, vol. 136, 103540, March 2021.

[17] T. Yoshida, Y. Tasaka, H. J. Park, Y. Murai, H. Teramura, and S. Koseki, "Inner Structure Visualization of Fresh Fruits Utilizing Ultrasonic Velocity Profiler,” Journal of Visualization, vol. 21, no. 2, pp. 253-265, February 2018.

[18] M. Aritomi, S. Zhou, M. Nakajima, Y. Takeda, M. Mori, and Y. Yoshioka, "Measurement System of Bubbly Flow Using Ultrasonic Velocity Profile Monitor and Video Data Processing Unit,” Journal of Nuclear Science and Technology, vol. 33, no. 12, pp. 915-923, 1996.

[19] Y. Suzuki, M. Nakagawa, M. Aritomi, H. Murakawa, H. Kikura, and M. Mori, "Microstructure of the Flow Field Around a Bubble in Counter-Current Bubbly Flow," Experimental Thermal and Fluid Science, vol. 26, no. 2-4, pp. 221-227, February 2002.

[20] H. Murakawa, H. Kikura, and M. Aritomi, "Application of Ultrasonic Multi-Wave Method for Two-Phase Bubbly and Slug Flows," Flow Measurement and Instrumentation, vol. 19, no. 3-4, pp. 205-213, June-August 2008.

[21] W. Wongsaroj, A. Hamdani, N. Thong-Un, H. Takahashi, and H. Kikura, "Extended Short-Time Fourier Transform for Ultrasonic Velocity Profiler on Two-Phase Bubbly Flow Using a Single Resonant Frequency,” Applied Sciences, vol. 9 , no. 1, 50, January 2019.

[22] Y. Takeda, Ultrasonic Doppler Velocity Profiler for Fluid Flow, New York: Springer, 2012.

[23] J. Krautkrämer and H. Krautkrämer, Ultrasonic Testing of Materials, 4th ed., Berlin: Springer, 1990.

[24] K. Tezuka, S. Wada, M. Mori, H. Kikura, and M. Aritomi, "Analysis of Ultrasound Propagation in a Steel Pipe Using Ultrasonic Pulse Doppler Method,” Thermal Science and Engineering, vol. 16, no. 3, pp. 95-104, April 2008.

Copyright $\subset$ by the authors. Licensee TAETI, Taiwan. This article is an open-access article distributed under the terms and conditions of the Creative Commons Attribution (CC BY-NC) license (https://creativecommons.org/licenses/by-nc/4.0/). 